University of Nebraska - Lincoln

DigitalCommons@University of Nebraska - Lincoln

4-18-2019

\title{
Nitrogen Fertilizer and Irrigation Effects on Seed Yield and Oil in Camelina
}

K. F. Bronson

USDA-ARS, U.S. Arid-Land Agricultural Research Center, kevin.bronson@ars.usda.gov

Douglas J. Hunsaker

USDA-ARS, U.S. Arid-Land Agricultural Research Center, doug.hunsaker@usda.gov

Kelly R. Thorp

USDA-ARS, U.S. Arid-Land Agricultural Research Center

Follow this and additional works at: https://digitalcommons.unl.edu/usdaarsfacpub

Bronson, K. F.; Hunsaker, Douglas J.; and Thorp, Kelly R., "Nitrogen Fertilizer and Irrigation Effects on Seed Yield and Oil in Camelina" (2019). Publications from USDA-ARS / UNL Faculty. 2140.

https://digitalcommons.unl.edu/usdaarsfacpub/2140

This Article is brought to you for free and open access by the U.S. Department of Agriculture: Agricultural Research Service, Lincoln, Nebraska at DigitalCommons@University of Nebraska - Lincoln. It has been accepted for inclusion in Publications from USDA-ARS / UNL Faculty by an authorized administrator of DigitalCommons@University of Nebraska - Lincoln. 


\title{
Nitrogen Fertilizer and Irrigation Effects on Seed Yield and Oil in Camelina
}

\author{
Kevin F. Bronson,* Douglas J. Hunsaker, and Kelly R. Thorp
}

\begin{abstract}
Interest is growing in camelina (Camelina sativa L. Crantz) as a biofuel feedstock. However, there has been little camelina research in irrigated arid systems. A 2-yr field study in Maricopa, AZ, under an overhead sprinkler irrigation system determined the effects of 10 water levels (irrigation fraction 0.5-1.1) and five $\mathrm{N}$ fertilizer rates $\left(38-150 \mathrm{~kg} \mathrm{~N} \mathrm{ha}^{-1}\right)$ on seed yield, seed oil content, and $\mathrm{N}$ use efficiency. Cultivar Robinson was planted in December 2012 and 2013. Nitrogen fertilizer (urea ammonium nitrate) was applied in three split applications. Irrigation amounts were from 125 to $380 \mathrm{~mm}$, and in-season rain was 70 and $50 \mathrm{~mm}$, in 2013 and 2014, respectively. Camelina seed yields were maximum at water level 7 (irrigation fraction $0.93)$ in 2013 at $1800 \mathrm{~kg} \mathrm{ha}^{-1}$. Maximum seed yields were 1600 $\mathrm{kg} \mathrm{ha}^{-1}$ at water level 6 (irrigation fraction 0.83 ) in 2014. These highest seed yields were achieved with $150 \mathrm{~kg} \mathrm{~N} \mathrm{ha}^{-1}$ in both years. Oil content (maximum 41\%) decreased with $\mathrm{N}$ rate but increased with water level. Seed $\mathrm{N}$ increased with $\mathrm{N}$ rate but decreased with irrigation level. Recovery efficiency of $\mathrm{N}$ fertilizer by camelina ranged from 12 to $72 \%$. The results indicate that good high-oil camelina yields can be produced in the southwestern United States with 320 to $380 \mathrm{~mm}$ irrigation plus rain and $\mathrm{N}$ fertilizer rates of $150 \mathrm{~kg} \mathrm{~N} \mathrm{ha}^{-1}$.
\end{abstract}

\section{Core Ideas}

- A 2-yr field study in Maricopa, AZ, under an overhead sprinkler irrigation system determined the effects of 10 water levels (irrigation fraction $0.5-1.1)$ and five $\mathrm{N}$ fertilizer rates $\left(38-150 \mathrm{~kg} \mathrm{~N} \mathrm{ha}^{-1}\right)$ on seed yield, seed oil, and $\mathrm{N}$ use efficiency.

- Recovery efficiency of $\mathrm{N}$ fertilizer by camelina ranged from 12 to $72 \%$ for high to low $\mathrm{N}$ fertilizer rates, respectively.

- Oil content of seed was maximum at $40 \%$, and decreased with $\mathrm{N}$ rate but increased with water level.

This document is a U.S. government work and is not subject to copyright in the United States.

Published in Agron. J. 111:1712-1719 (2019)

doi:10.2134/agronj2018.10.0644

Copyright (c) 2019 The author(s). Re-use requires permission from the publisher.
C

TAMELINA SATIVA is short-season, small-seeded oil crop of the Brassica family. It has been cultivated since 4000 BCE in southeastern Europe (Larsson, 2013), and was introduced to North America in the mid-1800s. There has been strong, renewed interest in camelina as a biofuel feedstock for biodiesel and jet fuel the last $15 \mathrm{yr}$ (Berti et al., 2016; Corporan et al., 2011; Sivakumar et al., 2015). Renewable jet fuel from camelina oil has been tested by the U.S. Air Force, KLM Royal Dutch, and Japan Airlines (Berti et al., 2016). The oil content of camelina seed is high, usually greater than 35\% (Zubr, 1997; Blackshaw et al., 2011; Mohammed et al., 2017). The oil profile of camelina is unique, with high amounts of unsaturated fatty acids, including 30 to $40 \%$ linolenic acid, 15 to $25 \%$ linoleic acid and about 15\% oleic acid (Zubr, 2003; Vollmann et al., 2007). Camelina is adapted to a very wide range of climatic conditions, including maritime Canada, the Plains of Canada, the American northern Plains, California, and Arizona (Gugel and Falk, 2006; Blackshaw et al., 2011; Berti et al., 2015; Aiken et al., 2015; Hunsaker et al., 2011) and reportedly can grow with lower $\mathrm{N}$ and water inputs than other oil seed crops like canola (Putnam et al., 1993; Budin et al., 1995).

Camelina is typically grown as a rainfed crop in Canada and the northern Great Plains. In the last 5 yr, there have been several published studies from Canada and the U.S. northern Plains on camelina production with irrigation (Hergert et al., 2016; Pavlista et al., 2016) and $\mathrm{N}$ and $S$ fertilizer management (Urbaniak et al., 2008; Jiang et al., 2013; Wysocki et al., 2013; Malhi et al., 2014; Mohammed et al., 2017). Irrigated camelina field studies in the American Southwest have been limited, where oil seed crops are typically not grown (French et al., 2009; Hunsaker et al., 2011, 2013). In general, the camelina irrigation studies have reported that seed yield increases with increasing amounts of water. However, none have presented specific guidance on the optimal irrigation water input needed to maximize water use efficiency (WUE; yield per unit water input; Pereira et al., 2012). Surprisingly, few studies have measured $N$ uptake and nitrogen use efficiency (NUE) in camelina (Malhi et al., 2014).

The multiple rainfed studies conducted showed a wide range of optimal $\mathrm{N}$ rates for camelina seed yields. Urbaniak et al. (2008) reported 60 to $80 \mathrm{~kg} \mathrm{~N} \mathrm{ha}^{-1}$ in the Maritime provinces of Canada. Jiang et al. (2013) published that optimal $N$ rates ranged

USDA-ARS, U.S. Arid Land Agricultural Research Center, 21881 N. Cardon Lane, Maricopa, AZ 85138. Received 5 Oct. 2018. Accepted 9 Jan. 2019. *Corresponding author (kevin.bronson@ars.usda.gov).

Abbreviations: AE, agronomic efficiency; ET, evapotranspiration; IUE, internal use nitrogen efficiency; NUE, nitrogen use efficiency; PE, physiological efficiency; RE, recovery efficiency; SDI, subsurface drip irrigation; TNU, total nitrogen uptake; WUE, water use efficiency. 
from 120 to $160 \mathrm{~kg} \mathrm{~N} \mathrm{ha}^{-1}$ in the Maritime Canada. Malhi et al. (2014) on the other hand, reported response of $\mathrm{N}$ fertilizer up to $200 \mathrm{~kg} \mathrm{~N} \mathrm{ha}^{-1}$ in Saskatchewan. Nitrogen fertilizer response data for camelina is lacking in irrigated arid regions. No studies combine tests of $\mathrm{N}$ and irrigation rates in camelina field trials.

The objectives of this study were (i) to determine the effects of $\mathrm{N}$ fertilizer rate on seed yield, aboveground biomass, total $\mathrm{N}$ uptake, $\mathrm{N}$ use efficiency, seed $\mathrm{N}$ content, and oil content at varying overhead sprinkler irrigation levels in an arid environment; and (ii) to estimate optimal $\mathrm{N}$ fertilizer rate and overhead sprinkler irrigation level for camelina seed yield, seed $\mathrm{N}$, and oil content.

\section{MATERIALS AND METHODS}

This camelina field experiment was performed in 2012-2013 and 2013-2014, on a 1.3-ha area in Maricopa, AZ. Mean annual rainfall is $190 \mathrm{~mm}$ and the soil type is Casa Grande sandy loam (fine-loamy, mixed, superactive, hyperthermic Typic Natrargid), that was $0.77 \%$ organic matter (Mon et al., 2016).

One 55-m long span of a two-span linear-move overhead irrigation system (Valmont Industries, Inc., Valmont, NE) was employed. Sprinklers were $1 \mathrm{~m}$ above the ground, and sprinklers were spaced $1.52 \mathrm{~m}$ apart. Pressure was maintained with a 69-kPa pressure regulator. Water was provided to the irrigation system through a $0.15 \mathrm{~m}$ diam. drag hose. Water came through a drag hose connected to a nearby pump that provided $1170 \mathrm{~L}$ $\mathrm{min}^{-1}$ of flow.

Scheduling of irrigations were linked to evapotranspiration $\left(E_{c}\right)$ estimates, calculated daily with FAO-56 dual crop coefficients (Allen et al., 1998; Mon et al., 2016). Seasonal basal crop coefficients $\left(K_{\mathrm{cb}}\right)$ used were from an empirically derived function based on camelina crop coefficient data obtained in prior field studies at this site (Hunsaker et al., 2013). A daily soil water balance estimated depletion of water in the camelina soil root zone, using irrigation and rainfall data.

A gradient of 10 non-randomized irrigation levels was imposed 1 mo after planting. Irrigation plot widths were $4.6 \mathrm{~m}$, with three sprinkler nozzles. The eighth irrigation level was considered the base irrigation 1.0 and the range in terms of irrigation fraction was 0.45 to 1.12 (Table 1). Irrigations were two times a week early in the growing season and were increased to three to four times a week during flowering. Seasonal total irrigation amounts for the 10 water levels in each year are presented in Table 1.

Soil samples were taken prior to planting at 36 points from 0 to $120-\mathrm{cm}$ deep. Soil profile $\mathrm{NO}_{3}-\mathrm{N}(0-100 \mathrm{~cm})$ was about 30 $\mathrm{kg} \mathrm{N} \mathrm{ha}^{-1}$ at the start of each season. Due to the low initial soil $\mathrm{NO}_{3}-\mathrm{N}$ levels, $30 \mathrm{~kg} \mathrm{~N} \mathrm{ha}^{-1}$ as ammonium sulfate was broadcast applied before final land preparation and planting. This starter $\mathrm{N}$ was not factored into the $\mathrm{N}$ treatment rates. Medium soil test $\mathrm{P}$ at the start of the study prompted us to broadcast and incorporate $39 \mathrm{~kg} \mathrm{ha}^{-1}$ of $\mathrm{P}_{2} \mathrm{O}_{5}$ as triple superphosphate.

Fifteen main experimental plots were established which were $90^{\circ}$ to the irrigation plots. The experimental design was a randomized complete block for $\mathrm{N}$ and there were three replicates. Nitrogen plots were 50 by $4 \mathrm{~m}$. Nitrogen fertilizer rates used were $0,38,75,113$, and $150 \mathrm{~kg} \mathrm{~N} \mathrm{ha}^{-1}$. The $\mathrm{N}$ source was urea ammonium nitrate $\left(320 \mathrm{~g} \mathrm{~N} \mathrm{~kg}^{-1}\right)$ which was applied with a sprayer-type tractor in three applications. Camelina cultivar Robinson was planted at $9 \mathrm{~kg}$ seed $\mathrm{ha}^{-1}$ in $15-\mathrm{cm}$ rows with a drill planter on 4 Dec. 2012 and 16 Dec. 2013.
Table I. Irrigation plus rain and the irrigation (plus rain) fractions based on irrigation level 8 in 20I2-2013 and 20I3-2014 (includes December 2012 and 20I3), Maricopa, AZ.

\begin{tabular}{|c|c|c|c|c|c|c|}
\hline \multirow{3}{*}{$\begin{array}{l}\text { Water } \\
\text { levels }\end{array}$} & \multicolumn{3}{|c|}{$2012-2013$} & \multicolumn{3}{|c|}{$2013-2014$} \\
\hline & \multicolumn{3}{|c|}{ Irrigation Irrigation } & \multicolumn{3}{|c|}{ Irrigation Irrigation } \\
\hline & Irrigation & + rain & fraction & Irrigation & + rain & fraction \\
\hline & \multicolumn{3}{|c|}{$\mathrm{mm}$} & \multicolumn{3}{|c|}{$\mathrm{mm}$} \\
\hline 1 & 127 & 196 & 0.49 & 125 & 174 & 0.45 \\
\hline 2 & 149 & 218 & 0.55 & 148 & 197 & 0.51 \\
\hline 3 & 174 & 243 & 0.61 & 174 & 223 & 0.58 \\
\hline 4 & 202 & 272 & 0.68 & 203 & 252 & 0.66 \\
\hline 5 & 234 & 303 & 0.76 & 236 & 284 & 0.74 \\
\hline 6 & 267 & 336 & 0.84 & 270 & 319 & 0.83 \\
\hline 7 & 306 & 375 & 0.94 & 310 & 359 & 0.93 \\
\hline 8 & 330 & 400 & 1.00 & 335 & 384 & 1.00 \\
\hline 9 & 350 & 420 & 1.05 & 356 & 405 & 1.05 \\
\hline 10 & 374 & 443 & I.II & 380 & 429 & 1.12 \\
\hline
\end{tabular}

Plant samples for biomass and seed were sampled by hand on 0.5-m sections of two rows for water levels $1,3,5,7$, and 9 at each $\mathrm{N}$ rate at maturity (22 Apr.-3 May 2013 and 25-29 Apr. 2014). Seed was threshed and screened; $\mathrm{N}$ content was determined on seed and stems on a Leco-Truspec CN analyzer (Leco Corp., St. Joseph, MO) after plant samples were dried at $70^{\circ} \mathrm{C}$ and milled to $0.5 \mathrm{~mm}$. Total nitrogen uptake (TNU) was calculated for seed and stems by multiplying plant $\mathrm{N}$ concentration by biomass. Seed oil content was analyzed with a TD-NMR minispectrometer (Model mq20, Bruker Optics, Inc., Billerica, MA). Water use efficiency for irrigation level was calculated as the ratio of seed yield to total water applied (irrigation plus rain).

Four NUE indices were calculated:

Recovery efficiency (RE) was calculated as (Dilz, 1988):

$$
\operatorname{RE}(\%)=\frac{(\text { TNU in N fertilized plot }- \text { TNU in Zero }-\mathrm{N} \text { plot }) \times 100}{\mathrm{~N} \text { fertilizer rate in } \mathrm{N}-\text { fertilized plot }}
$$

where TNU $=$ total $\mathrm{N}$ accumulation in seed and stems.

Physiological efficiency (PE) was calculated as (Isfan, 1990):

$$
\begin{aligned}
& \mathrm{PE}(\mathrm{kg} \text { seed } / \mathrm{kg} \mathrm{N} \text { in plant })= \\
& \frac{\text { Seed yield in } \mathrm{N} \text { fertilized plot }- \text { seed yield in Zero }-\mathrm{N} \text { plot }}{\text { TNU in } \mathrm{N}-\text { fertilized plot }-\mathrm{TNU} \text { in Zero }-\mathrm{N} \text { plot }}
\end{aligned}
$$

Agronomic efficiency (AE) was calculated as (Novoa and Loomis, 1981):

$$
\begin{aligned}
& \mathrm{AE}(\mathrm{kg} \text { seed } / \mathrm{kg} \mathrm{N} \text { applied })= \\
& \frac{\text { Seed yield in } \mathrm{N} \text { fertilized plot }- \text { seed yield in Zero }-\mathrm{N} \text { plot }}{\mathrm{N} \text { fertilizer rate in fertilized plot }}
\end{aligned}
$$

Internal use nitrogen efficiency (IUE) was determined by the ratio of seed yield and total $\mathrm{N}$ accumulation (Witt et al., 1999).

The fixed effects of $\mathrm{N}$, irrigation level, and $\mathrm{N} \times$ water interaction on seed yield, WUE, seed N, seed oil, biomass, TNU, RE, PE, and IUE were estimated with SAS PROC MIXED procedures (SAS Institute, 2013). Irrigation level was considered a repeated measure as suggested by Piepho et al. (2004) when irrigation levels are not replicated to account for the correlations arising from serial ordering of irrigation levels. Replicate and replicate by $\mathrm{N}$ rate were treated as random effects. Additionally, a PROC MIXED procedure was performed by water level, with just $\mathrm{N}$ rate as a fixed effect. 
Table 2. The ANOVA $F$ tests of $N$ fertilizer rate, Water level, and $N \times$ water interaction on camelina total biomass, total $N$ uptake, seed yield, water use efficiency (WUE), seed N, oil content, oil yield, recovery efficiency of nitrogen (RE), physiological nitrogen use efficiency (PE), internal nitrogen use efficiency (IUE), and agronomic nitrogen use efficiency (AE), in 20I3, and 20I4, Maricopa, AZ.

\begin{tabular}{|c|c|c|c|c|c|c|c|c|c|c|c|}
\hline$F$ test & Total biomass & s Total N uptake & Seed yield & WUE & Seed N & Oil content & Oil yield & RE & $\mathrm{PE}$ & IUE & $\mathrm{AE}$ \\
\hline & \multicolumn{11}{|c|}{2013} \\
\hline $\mathrm{N}$ rate & $13^{* *}$ & $72^{* *}$ & $52 * *$ & $27^{* *}$ & $1 \mathrm{II} * *$ & $19 *$ & $44 * *$ & $3.9 *$ & $5.5^{*}$ & $37^{* *}$ & $6.1 * *$ \\
\hline Water & $5.2^{* *}$ & $8.2 * *$ & $23 * *$ & $14 * *$ & $62 * *$ & $51 *$ & $32 * *$ & II** & $10 * *$ & $39 * *$ & $20 * *$ \\
\hline \multirow[t]{2}{*}{$\mathrm{N} \times$ water } & nst & $3.6 * *$ & $4.8^{* *}$ & $2.5^{* *}$ & ns & ns & $5.2^{* *}$ & ns & ns & ns & $2.0 *$ \\
\hline & \multicolumn{11}{|c|}{2014} \\
\hline $\mathrm{N}$ rate & $6.1 * *$ & $26 * *$ & $15^{* *}$ & $15 * *$ & $14.8^{* *}$ & $27 * *$ & $9.3^{* *}$ & ns & $8.6^{*}$ & $10^{* *}$ & ns \\
\hline Water & $2.8^{*}$ & $5.6 * *$ & $10 * *$ & $20 * *$ & $2.5^{*}$ & $25 * *$ & $12.5 * *$ & $5.1 * *$ & $5 . I^{* *}$ & $24 * *$ & $2.7^{*}$ \\
\hline $\mathrm{N} \times$ water & ns & ns & ns & ns & ns & ns & ns & ns & ns & $1.7 *$ & ns \\
\hline
\end{tabular}

* Significant at $P<0.05$.

** Significant at $P<0.01$.

$\dagger$ ns is not significant at $P=0.05$.

\section{RESULTS}

Plant biomass increased in a significant, linear fashion with irrigation level in 2013 and in 2014 (Tables 2 and 3). Biomass ranged from 4 to $11 \mathrm{Mg} \mathrm{ha}^{-1}$. Plant biomass in 2014 was slightly higher than in 2013. Maximum biomass yields were $9.5 \mathrm{Mg}$ $\mathrm{ha}^{-1}$ in 2013 at water level 9 (irrigation fraction 1.05) with $150 \mathrm{~kg} \mathrm{~N} \mathrm{ha}^{-1}$ and $11.1 \mathrm{Mg} \mathrm{ha}^{-1}$ in 2014 at water level 5 (irrigation fraction 0.74 ) with $113 \mathrm{~kg} \mathrm{~N} \mathrm{ha}^{-1}$. Nitrogen fertilizer rate significantly affected biomass in both years at water levels 1 to 7 (Table 3). Biomass had no water $\times \mathrm{N}$ interaction.

Camelina TNU responded to water levels in both seasons (Table 2). A positive $\mathrm{N}$ rate effect on TNU was observed at all irrigation levels in both growing seasons, except for water level one in 2013 (Table 3). The maximum TNU was $100 \mathrm{~kg} \mathrm{~N} \mathrm{ha}^{-1}$ in 2013 at water level 7 with $150 \mathrm{~kg} \mathrm{~N} \mathrm{ha}^{-1}$ applied and $103 \mathrm{~kg}$ $\mathrm{N} \mathrm{ha}^{-1}$ in 2014 at water level 3 with $113 \mathrm{~kg} \mathrm{~N} \mathrm{ha}^{-1}$ applied. With zero-N TNU was not affected by water level in 2013 or 2014 (data not shown).

Seed yield exhibited a quadratic response to irrigation in 2013 at all $\mathrm{N}$ rates (Fig. 1). The optimal irrigation level (i.e., yield maximizing level) increased with $\mathrm{N}$ fertilizer rate. The optimal irrigation level decreased to 0.68 irrigation fraction at zero N. A maximum seed yield of $1800 \mathrm{~kg} \mathrm{ha}^{-1}$ was achieved at $150 \mathrm{~kg} \mathrm{~N} \mathrm{ha}^{-1}$ at irrigation level 7 (fraction 0.93) in 2013 (Fig. 1). Seed yield was greatest at $150 \mathrm{~kg} \mathrm{~N} \mathrm{ha}^{-1}$ at all irrigation levels greater than level three (0.61 fraction) (Fig. 2). The optimal $\mathrm{N}$ fertilizer rate decreased to $75 \mathrm{~kg} \mathrm{~N} \mathrm{ha}^{-1}$ at the third irrigation level (fraction 0.61), and at irrigation level one there was no $\mathrm{N}$ rate response (Fig. 2).

In 2014, bird damage was significant in water levels 7 to 10, therefore we are not showing yield data for those water levels. Seed yields were lower in 2014 at the medium to high levels of water and $\mathrm{N}$ fertilizer (Fig. 1). Similar to 2013, the optimal irrigation level was at the highest $\mathrm{N}$ rate (Fig. 1). Maximum seed yield was $1600 \mathrm{~kg}$ $\mathrm{ha}^{-1}$ in season 2014 at $150 \mathrm{~kg} \mathrm{~N} \mathrm{ha}^{-1}$ and at irrigation level $6(0.83$ irrigation fraction). The optimal $\mathrm{N}$ fertilizer rate at irrigation levels 1 to 5 in 2014 was $113 \mathrm{~kg} \mathrm{~N} \mathrm{ha}^{-1}$ (Fig. 2). The optimal irrigation fraction at zero $\mathrm{N}$ and $38 \mathrm{~kg} \mathrm{~N} \mathrm{ha}^{-1}$ in 2014 was 0.65 (Fig. 2).

Water use efficiencies decreased in a quadratic manner with irrigation level at all $\mathrm{N}$ rates (Fig. 3). A maximum WUE of 0.54 was observed in 2013 at $150 \mathrm{~kg} \mathrm{~N} \mathrm{ha}^{-1}$ at water level 5 (fraction $0.76)$, two levels less than the yield maximizing irrigation level (Fig. 3 and 4). Greater WUEs were observed with increasing N rate, varying from a low of $<0.1 \mathrm{~kg} \mathrm{~m}^{-3}$ at water level 10 with zero $\mathrm{N}$ in 2013 to $0.54 \mathrm{~kg} \mathrm{~m}^{-3}$ at water level 3 and $150 \mathrm{~kg} \mathrm{~N}$ $\mathrm{ha}^{-1}$ in 2013 (Fig. 4). There were positive quadratic responses of WUE to $\mathrm{N}$ rate at all irrigation levels (except 0.49 irrigation fraction in 2013) in both years (Table 2, Fig. 4). A maximum WUE of $0.65 \mathrm{~kg} \mathrm{~m}^{-3}$ was observed in 2014 at $113 \mathrm{~kg} \mathrm{~N} \mathrm{ha}^{-1}$ at water level 3 (fraction 0.61), three levels less than the yield maximizing irrigation level (Fig. 3 and 4).

Seed $N$ responded negatively to irrigation level in 2013 and 2014 (Table 3). A highly significant effect of $\mathrm{N}$ rate $(P<0.01)$ was observed at every water level in both years (Table 2)

Seed oil content responded positively to irrigation level in both seasons, and negatively to $\mathrm{N}$ fertilizer rate (Table 3). Maximum seed oil content was 41\% in 2013 and 40\% in 2014. On average, seed oil content was lower in Year 2. The negative relation between $\mathrm{N}$ rate and seed oil content was significant at $P<0.05$ and at all irrigation levels in both years. However, when oil yields per hectare are calculated, the responses to $\mathrm{N}$ fertilizer and irrigation corresponded closely with seed yields. The highest oil yields were 740 and $600 \mathrm{~kg} \mathrm{ha}^{-1}$ in 2013, and 2014, respectively (data not shown).

Nitrogen RE generally increased with irrigation level in both seasons (Tables 2 and 3). Nitrogen rate interacted in a significant negative linear trend with RE in two of five water levels in 2013 only. The magnitude of RE was less in 2014, with a maximum of $72 \%$ in 2013 and $58 \%$ in 2013, especially at water levels 7 and 9 (Table 3).

Physiological use efficiency and IUE were similar between the two, seasons, except for water level 7, where they were lower in the second season (Table 3). The PE and IUE increased with irrigation level. Nitrogen fertilizer effected RE, PE, IUE, and $\mathrm{AE}$ in a negative manner, but only at some of the water levels, more so in season one (Table 3). Negative linear contrasts were significant for $\mathrm{AE}$ at two of five water levels in both years. Internal use efficiency had significant negative linear trends with $\mathrm{N}$ rate in three of five water levels in 2013 and in two in 2014 (Table 3). Agronomic efficiency is the product of RE and $\mathrm{PE}$, and was therefore also reduced in 2014, compared to 2013 in water levels 7 and 9 (Table 3).

\section{DISCUSSION}

The high camelina seed yields in this study were similar to the 1500 to $1600 \mathrm{~kg} \mathrm{ha}^{-1}$ reported by Hunsaker et al. (2013) with cultivar Robinson. However, the yields we report here are 25 to $50 \%$ lower than the 2500 to $2900 \mathrm{~kg} \mathrm{ha}^{-1}$ reported with overhead sprinkler irrigation in Nebraska (Pavlista et al., 2016; 
Table 3. Camelina biomass $\left(\mathrm{Mg} \mathrm{ha}{ }^{-1}\right)$, total $\mathrm{N}$ uptake $\left(\mathrm{kg} \mathrm{N} \mathrm{ha}^{-1}\right)$, recovery efficiency of nitrogen (RE, \%), seed $\mathrm{N}(\%)$, and oil content (\%) of camelina as affected by $\mathrm{N}$ fertilizer rate and water level, in 20I3, and 20I4, Maricopa, AZ.

\begin{tabular}{|c|c|c|c|c|c|c|c|c|c|c|c|}
\hline \multirow{3}{*}{$\begin{array}{l}\text { Water } \\
\text { level }\end{array}$} & \multirow{3}{*}{$\begin{array}{c}\text { Dependent } \\
\text { variable } \dagger\end{array}$} & \multicolumn{10}{|c|}{$\mathrm{N}$ fertilizer rate, $\mathrm{kg} \mathrm{N} \mathrm{ha}^{-\mathrm{I}}$} \\
\hline & & 38 & 75 & 113 & 150 & \multirow[b]{2}{*}{ SE } & 38 & 75 & 113 & 150 & \multirow[b]{2}{*}{ SE } \\
\hline & & \multicolumn{4}{|c|}{2013} & & \multicolumn{4}{|c|}{2014} & \\
\hline \multirow[t]{24}{*}{ I } & Biomass & 4.4 & 4.5 & 5.3 & 6.0 & 0.7 & 6.5 & 6.9 & 6.7 & 8.9 & 0.6 \\
\hline & Linear & $*$ & & & & & $*$ & & & & \\
\hline & Quadratic & nsł & & & & & ns & & & & \\
\hline & $\mathrm{N}$ uptake & 50 & 52 & 58 & 48 & 7.2 & 52 & 57 & 75 & 75 & 5.9 \\
\hline & Linear & ns & & & & & $* *$ & & & & \\
\hline & Quadratic & ns & & & & & ns & & & & \\
\hline & RE & 31 & 18 & 32 & 16 & II & 42 & 28 & 34 & 26 & 6.8 \\
\hline & Linear & ns & & & & & ns & & & & \\
\hline & Quadratic & ns & & & & & ns & & & & \\
\hline & $\mathrm{AE}$ & 3.7 & 2.1 & 1.6 & 0 & 2.0 & 6.5 & 4.7 & 3.2 & 2.7 & 1.0 \\
\hline & Linear & ns & & & & & $*$ & & & & \\
\hline & Quadratic & ns & & & & & ns & & & & \\
\hline & PE & 9.1 & 12.8 & 10.8 & 0 & 7.0 & 15.3 & 19.0 & 9.2 & 10.5 & 2.5 \\
\hline & Linear & ns & & & & & ns & & & & \\
\hline & Quadratic & ns & & & & & ns & & & & \\
\hline & IUE & 14.9 & 15.1 & 13.6 & 13.1 & 1.4 & 15.5 & 16.3 & 12.4 & 13.0 & 0.8 \\
\hline & Linear & ns & & & & & $*$ & & & & \\
\hline & Quadratic & ns & & & & & $*$ & & & & \\
\hline & Seed N & 4.5 & 4.6 & 4.7 & 4.8 & 0.1 & 3.9 & 4.2 & 4.4 & 4.4 & 0.1 \\
\hline & Linear & $* *$ & & & & & $* *$ & & & & \\
\hline & Quadratic & ns & & & & & ns & & & & \\
\hline & Oil content & 34 & 34 & 32 & 32 & 0.6 & 33 & 34 & 32 & 30 & 0.8 \\
\hline & Linear & $*$ & & & & & $* *$ & & & & \\
\hline & Quadratic & ns & & & & & ns & & & & \\
\hline \multirow[t]{24}{*}{3} & Biomass & 4.7 & 4.5 & 4.6 & 5.6 & 0.5 & 8.2 & 7.5 & 8.0 & 9.0 & 0.7 \\
\hline & Linear & $* *$ & & & & & $*$ & & & & \\
\hline & Quadratic & ns & & & & & ns & & & & \\
\hline & $\mathrm{N}$ uptake & 53 & 57 & 58 & 59 & 3.0 & 60 & 71 & 103 & 93 & 5.6 \\
\hline & Linear & $* *$ & & & & & $* *$ & & & & \\
\hline & Quadratic & $*$ & & & & & ns & & & & \\
\hline & RE & 42 & 26 & 18 & 15 & 3.2 & 58 & 44 & 57 & 36 & 11 \\
\hline & Linear & $* *$ & & & & & ns & & & & \\
\hline & Quadratic & ns & & & & & ns & & & & \\
\hline & $\mathrm{AE}$ & 5.0 & 2.9 & 2.0 & 1.2 & 0.5 & 9.2 & 6.5 & 5.8 & 3.9 & 2.0 \\
\hline & Linear & $* *$ & & & & & $*$ & & & & \\
\hline & Quadratic & ns & & & & & ns & & & & \\
\hline & PE & 12.3 & 10.9 & II.I & 7.4 & 1.8 & 15.8 & 13.8 & 10.1 & 11.0 & 1.7 \\
\hline & Linear & $*$ & & & & & $*$ & & & & \\
\hline & Quadratic & ns & & & & & ns & & & & \\
\hline & IUE & 17.7 & 16.4 & 16.4 & 15.1 & 0.6 & 19.0 & 17.8 & 14.0 & 14.9 & 0.9 \\
\hline & Linear & $* *$ & & & & & $* *$ & & & & \\
\hline & Quadratic & ns & & & & & ns & & & & \\
\hline & Seed N & 4.1 & 4.5 & 4.4 & 4.7 & 0.1 & 3.9 & 4.2 & 4.4 & 4.5 & 0.1 \\
\hline & Linear & $* *$ & & & & & $* *$ & & & & \\
\hline & Quadratic & ns & & & & & ns & & & & \\
\hline & Oil content & 37 & 35 & 35 & 34 & 0.7 & 38 & 36 & 35 & 35 & 0.4 \\
\hline & Linear & $* *$ & & & & & $* *$ & & & & \\
\hline & Quadratic & ns & & & & & ns & & & & \\
\hline \multirow[t]{5}{*}{5} & Biomass & 5.2 & 5.9 & 5.6 & 6.8 & 0.6 & 7.7 & 8.4 & II.I & 10.2 & 1.2 \\
\hline & Linear & $* *$ & & & & & $*$ & & & & \\
\hline & Quadratic & ns & & & & & ns & & & & \\
\hline & $\mathrm{N}$ uptake & 47 & 63 & 79 & 94 & 4.1 & 56 & 71 & 81 & 83 & 6.0 \\
\hline & Linear & $* *$ & & & & & $* *$ & & & & \\
\hline
\end{tabular}




\begin{tabular}{|c|c|c|c|c|c|c|c|c|c|c|c|}
\hline \multirow{3}{*}{$\begin{array}{l}\text { Water } \\
\text { level }\end{array}$} & \multirow{3}{*}{$\begin{array}{c}\text { Dependent } \\
\text { variablet }\end{array}$} & \multicolumn{10}{|c|}{$\mathrm{N}$ fertilizer rate, $\mathrm{kg} \mathrm{N} \mathrm{ha}^{-\mathrm{I}}$} \\
\hline & & 38 & 75 & 113 & 150 & \multirow[b]{2}{*}{ SE } & 38 & 75 & 113 & 150 & \multirow[b]{2}{*}{ SE } \\
\hline & & \multicolumn{4}{|c|}{2013} & & \multicolumn{4}{|c|}{2014} & \\
\hline & Quadratic & ns & & & & & ns & & & & \\
\hline & RE & 47 & 45 & 44 & 43 & 5.9 & 44 & 42 & 37 & 29 & 12 \\
\hline & Linear & ns & & & & & ns & & & & \\
\hline & Quadratic & ns & & & & & ns & & & & \\
\hline & $A E$ & 9.1 & 7.8 & 7.0 & 6.1 & I.I & 11.3 & 8.1 & 5.2 & 4.7 & 2.3 \\
\hline & Linear & ns & & & & & ns & & & & \\
\hline & Quadratic & ns & & & & & ns & & & & \\
\hline & PE & 19.4 & 16.7 & 16.0 & 14.1 & 0.4 & 37.8 & 20.0 & 14.1 & 15.9 & 7.3 \\
\hline & Linear & $* *$ & & & & & $*$ & & & & \\
\hline & Quadratic & ns & & & & & ns & & & & \\
\hline & IUE & 22.2 & 20.1 & 18.9 & 17.2 & 0.5 & 20.5 & 18.7 & 16.1 & 17.0 & 1.0 \\
\hline & Linear & $* *$ & & & & & ns & & & & \\
\hline & Quadratic & ns & & & & & $*$ & & & & \\
\hline & Seed N & 3.6 & 4.0 & 4.3 & 4.5 & 0.03 & 3.9 & 4.5 & 4.7 & 4.7 & 0.1 \\
\hline & Linear & $* *$ & & & & & $* *$ & & & & \\
\hline & Quadratic & ns & & & & & $* *$ & & & & \\
\hline & Oil content & 40 & 37 & 36 & 35 & 0.7 & 39 & 36 & 35 & 35 & 0.8 \\
\hline & Linear & $* *$ & & & & & $* *$ & & & & \\
\hline & Quadratic & ns & & & & & ns & & & & \\
\hline \multirow[t]{24}{*}{7} & Biomass & 5.4 & 5.8 & 7.4 & 8.4 & 0.8 & 7.1 & 9.0 & 9.2 & 7.5 & 1.3 \\
\hline & Linear & $* *$ & & & & & ns & & & & \\
\hline & Quadratic & $* *$ & & & & & ns & & & & \\
\hline & $\mathrm{N}$ uptake & 4011 & 71 & 77 & 100 & 2.8 & 45 & 56 & 70 & 56 & 8.6 \\
\hline & Linear & $* *$ & & & & & $*$ & & & & \\
\hline & Quadratic & ns & & & & & ns & & & & \\
\hline & RE & 37 & 60 & 45 & 49 & 3.2 & 17 & 23 & 28 & 12 & 9.8 \\
\hline & Linear & ns & & & & & ns & & & & \\
\hline & Quadratic & $*$ & & & & & ns & & & & \\
\hline & $A E$ & 8.0 & 10.8 & 8.2 & 7.9 & 0.7 & 3.5 & 2.7 & 3.9 & 1.6 & 2.1 \\
\hline & Linear & ns & & & & & ns & & & & \\
\hline & Quadratic & ns & & & & & ns & & & & \\
\hline & PE & 21.4 & 18.2 & 18.2 & 16.0 & 1.6 & 35.2 & 6.4 & 7.8 & 12.3 & 10.1 \\
\hline & Linear & $*$ & & & & & ns & & & & \\
\hline & Quadratic & & & & & & ns & & & & \\
\hline & IUE & 22.8 & 20.1 & 20.0 & 18.0 & 0.7 & 17.6 & 15.0 & 15.1 & 15.8 & I.I \\
\hline & Linear & $* *$ & & & & & ns & & & & \\
\hline & Quadratic & ns & & & & & ns & & & & \\
\hline & Seed $N$ & 3.4 & 3.7 & 4.0 & 4.2 & 0.05 & 4.2 & 4.6 & 4.7 & 4.7 & 0.1 \\
\hline & Linear & $* *$ & & & & & $* *$ & & & & \\
\hline & Quadratic & ns & & & & & $*$ & & & & \\
\hline & Oil content & 40 & 40 & 39 & 38 & 0.6 & 37 & 36 & 35 & 35 & 1.0 \\
\hline & Linear & $*$ & & & & & $* *$ & & & & \\
\hline & Quadratic & ns & & & & & ns & & & & \\
\hline \multirow[t]{11}{*}{9} & Biomass & 6.2 & 6.7 & 7.1 & 9.5 & I.I & 8.7 & 9.5 & 10.4 & 9.4 & 1.9 \\
\hline & Linear & $* *$ & & & & & ns & & & & \\
\hline & Quadratic & ns & & & & & ns & & & & \\
\hline & $\mathrm{N}$ uptake & 41 & 53 & 63 & 84 & 4.3 & 46 & 63 & 75 & 77 & 6.0 \\
\hline & Linear & $* *$ & & & & & $* *$ & & & & \\
\hline & Quadratic & ns & & & & & ns & & & & \\
\hline & RE & 72 & 53 & 44 & 47 & 5.1 & 38 & 30 & 31 & 24 & 9.0 \\
\hline & Linear & $* *$ & & & & & ns & & & & \\
\hline & Quadratic & ns & & & & & ns & & & & \\
\hline & $\mathrm{AE}$ & 16.6 & 10.8 & 8.9 & 8.9 & I.I & 4.6 & 7.3 & 5.3 & 4.2 & 3.7 \\
\hline & Linear & $* *$ & & & & & ns & & & & \\
\hline
\end{tabular}




\begin{tabular}{|c|c|c|c|c|c|c|c|c|c|c|c|}
\hline \multirow{3}{*}{$\begin{array}{l}\text { Water } \\
\text { level }\end{array}$} & \multirow{3}{*}{$\begin{array}{l}\text { Dependent } \\
\text { variable } \dagger\end{array}$} & \multicolumn{10}{|c|}{$\mathrm{N}$ fertilizer rate, $\mathrm{kg} \mathrm{N} \mathrm{ha}^{-\mathrm{I}}$} \\
\hline & & 38 & 75 & 113 & 150 & \multirow[b]{2}{*}{ SE } & 38 & 75 & 113 & 150 & \multirow[b]{2}{*}{ SE } \\
\hline & & \multicolumn{4}{|c|}{2013} & & \multicolumn{4}{|c|}{2014} & \\
\hline & Quadratic & $*$ & & & & & ns & & & & \\
\hline & PE & 23.1 & 20.3 & 20.5 & 19.1 & 0.7 & 40.9 & 24.4 & 17.1 & 17.6 & 5.3 \\
\hline & Linear & $* *$ & & & & & $*$ & & & & \\
\hline & Quadratic & ns & & & & & ns & & & & \\
\hline & IUE & 22.4 & 20.5 & 20.5 & 19.4 & 0.7 & 19.9 & 20.9 & 18.2 & 18.3 & 1.2 \\
\hline & Linear & ns & & & & & ns & & & & \\
\hline & Quadratic & ns & & & & & ns & & & & \\
\hline & Seed N & 3.4 & 3.6 & 3.8 & 4.0 & 0.03 & 4.0 & 4.4 & 4.6 & 4.4 & 0.2 \\
\hline & Linear & $* *$ & & & & & $* *$ & & & & \\
\hline & Quadratic & ns & & & & & ns & & & & \\
\hline & Oil content & 41 & 40 & 40 & 40 & 0.4 & 40 & 37 & 36 & 36 & 0.4 \\
\hline & Linear & $*$ & & & & & $* *$ & & & & \\
\hline & Quadratic & ns & & & & & ns & & & & \\
\hline
\end{tabular}

* Significant at $P<0.05$.

** Significant at $P<0.01$.

† $A E$, agronomic efficiency; $P E$, physiological efficiency; IUE, internal use nitrogen efficiency.

$\ddagger$ ns is not significant at $P=0.05$.
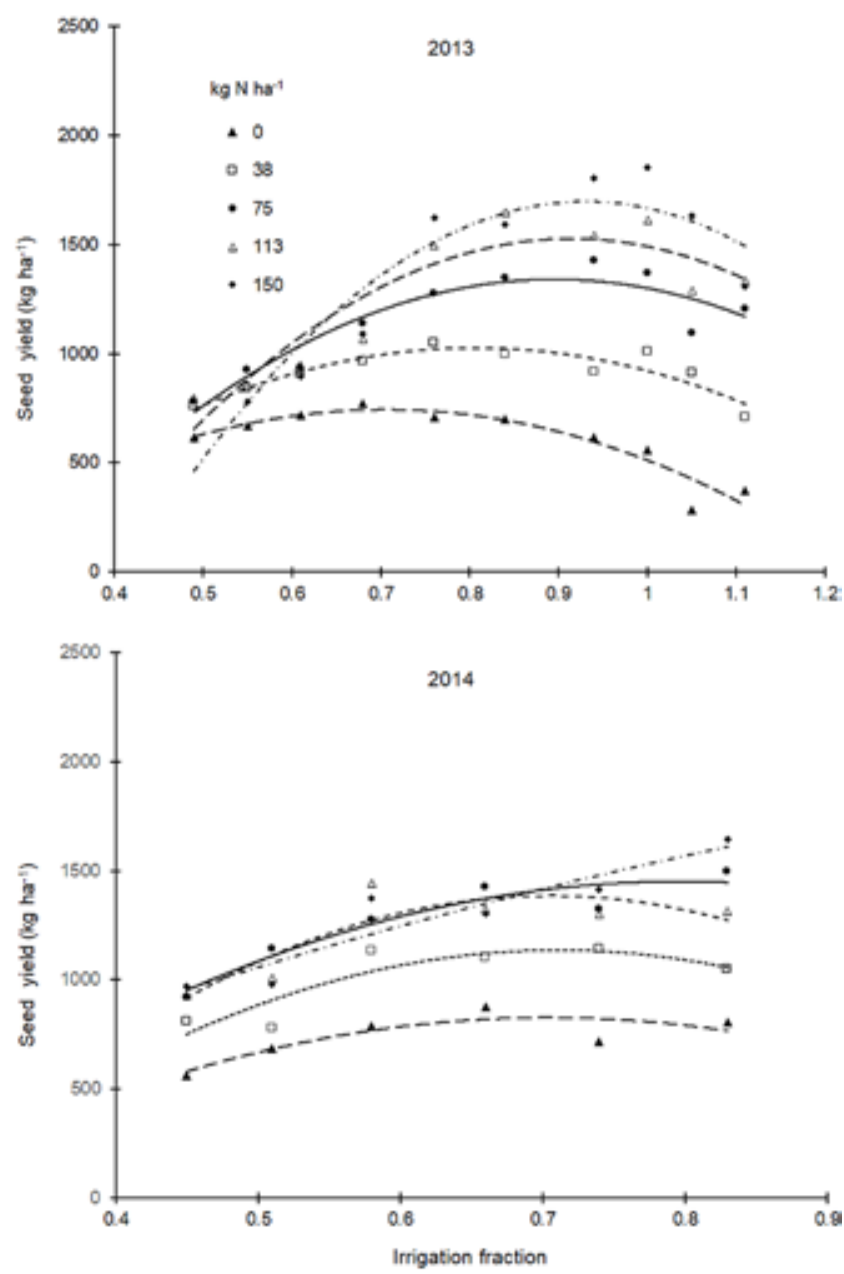

Fig. I. Seed yield of camelina as influenced by water level and $\mathrm{N}$ rates, 2013, and 2014, Maricopa, AZ.
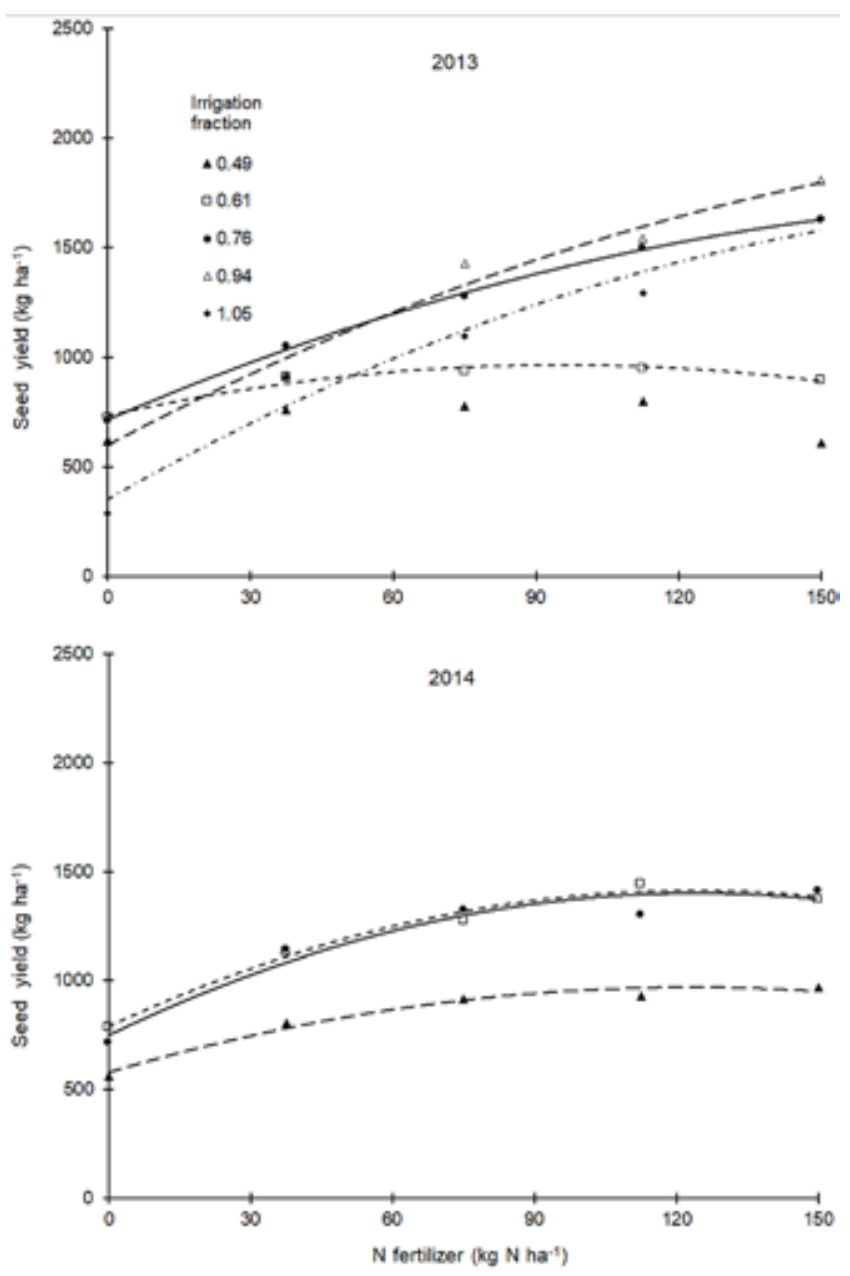

Fig. 2. Seed yield of camelina as influenced by $\mathrm{N}$ rate at different water levels, 2013, and 2014, Maricopa, AZ. 

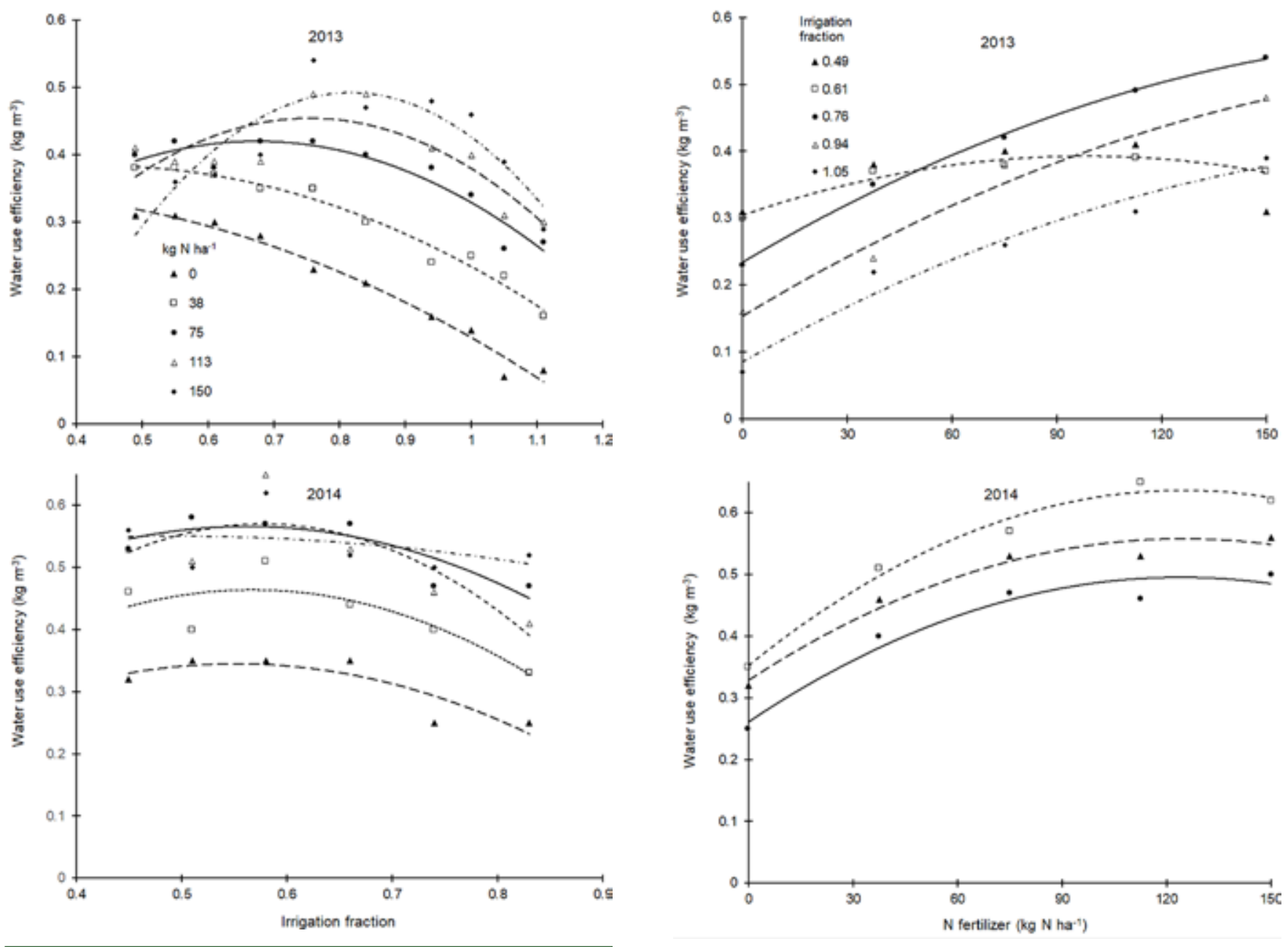

Fig. 3. Water use efficiency of camelina as influenced by water level at different $\mathrm{N}$ rates, 20I3, and 20I4, Maricopa, AZ.

Hergert et al., 2016), with similarly high irrigation levels of $>300 \mathrm{~mm}$. The Nebraska studies had more rain than in Arizona, the soils were higher in organic matter, and a different culti$\operatorname{var}$ (Cheyenne) was used. The irrigation amounts we applied were about one-third less than in a companion durum wheat (Triticum durum Desf.) study (Mon et al., 2016). Lower seed yields in 2014 can probably be attributed to a markedly warmer winter (January and February), resulting in earlier maturity compared to 2013. Additionally, germination rates in Year 2 were lower than in Year 1. However, biomass and total $\mathrm{N}$ uptake were similar between the $2 \mathrm{yr}$. The response of seed yields to $\mathrm{N}$ rate revealed that $150 \mathrm{~kg} \mathrm{~N} \mathrm{ha}^{-1}$ was required in both years.

Few camelina field studies report $\mathrm{N}$ uptake. The $\mathrm{N}$ recovery efficiency we report was similar to the maximum RE reported by Malhi et al. (2014). Lower RE and AE at water levels 7 and 9 in 2014 compared to 2013 reflect the warm spring of 2014. This was in conjunction with similar biomass and $\mathrm{N}$ uptake between the two seasons.

Water use efficiencies observed here under a large number of overhead sprinkler irrigation levels had a wider range than those reported by Hunsaker et al. (2013) with surface irrigation. Maximum WUE with overhead sprinkler $\left(0.54-0.65 \mathrm{~kg} \mathrm{~m}^{-3}\right)$ was higher than the $0.35 \mathrm{~kg} \mathrm{~m}^{-3}$ under surface irrigation. Optimal irrigation level for maximum WUE was several levels lower than the water levels that corresponded with maximum seed yield in 2013

and 2014. Optimal N rates for maximum WUE corresponded with the optimal $\mathrm{N}$ rates for seed yield in both seasons.

The optimal seed yield response to water appeared fairly consistent between the $2 \mathrm{yr}$ at level 6 to 7 , which translated to 320 to $376 \mathrm{~mm}$ irrigation plus rain. The optimal $\mathrm{N}$ rate was 150 $\mathrm{kg} \mathrm{N} \mathrm{ha}{ }^{-1}$ in both years. Both water and $\mathrm{N}$ are needed at the mid- to high ranges of the rates tested and both inputs are critical to maximize yields. Large yield reductions were observed at the low end of the $\mathrm{N}$ and/or water levels applied.

The effects of water and $\mathrm{N}$ rate on seed $\mathrm{N}$ and oil content were the most consistent of the dependent variables measured, with significant effects detected for every irrigation level and for both years. Seed oil content was similar between the two seasons and consistent with most published studies (French et al., 2009; Jiang et al., 2013; Wysocki et al., 2013; Malhi et al., 2014). Oil content showed a strong positive response to irrigation level and a negative relation with $\mathrm{N}$ rate. However, as indicated above the choice of optimum $\mathrm{N}$ fertilizer and irrigation levels for seed oil yields should be based on simply optimizing seed yields. Seed N content had the opposite trend of seed oil content, being negative with water and positive with $\mathrm{N}$ rate. These trends were also reported in rainfed studies in the Canadian prairies (Jiang et al., 2013; Malhi et al., 2014) and in maritime Canada (Urbaniak et al., 2008). On the other hand, rainfed studies in Washington and Montana reported no effect of $\mathrm{N}$ rate on camelina oil content (Wysocki et 
al., 2013; Mohammed et al., 2017). Seed N effects of N fertilizer rate and irrigation level were similar to published studies with rainfed camelina (Jiang et al., 2013; Malhi et al., 2014).

\section{CONCLUSION}

This $\mathrm{N} \times$ water camelina study showed that in a desert environment, strong relationships exists between seed yield, seed $\mathrm{N}$, seed oil, fertilizer $\mathrm{N}$ rate, and irrigation level. The camelina cultivar Robinson responded strongly to irrigation level and $\mathrm{N}$ fertilizer rate, though a warmer second season resulted in lower than expected yields. The optimal irrigation level (0.93 fraction) for maximum camelina seed yield was nearly 1.0 base irrigation level at high $\mathrm{N}$ rates in 2012-2013, which had near-normal temperatures and high yields. In the second season, which had warmer temperatures and lower yields, the sixth irrigation level $(0.83$ fraction) was the optimum for maximum yields. The optimum $\mathrm{N}$ fertilizer rate was $150 \mathrm{~kg} \mathrm{~N} \mathrm{ha}^{-1}$ rate in both seasons. Camelina growers can produce the highest yield and seed oil by applying 150 $\mathrm{kg} \mathrm{N} \mathrm{ha}^{-1}$ and keeping depletion of soil water $<45 \%$ at the 0.83 to 0.94 base irrigation level. These results indicate the potential for camelina production in other irrigated, arid regions of the world.

\section{REFERENCES}

Aiken, R., D. Baltensperger, J. Krall, A. Pavlista, and J. Johnson. 2015. Planting methods affect emergence, flowering and yield of spring oilseed crops in the US central High Plains. Ind. Crops Prod. 69:273-277. doi:10.1016/j. indcrop.2015.02.025

Allen, R.G., L.S. Pereira, D. Raes, and M. Smith. 1998. Crop Evapotranspiration. FAO Irrigation and Drainage Paper 56. Food and Agriculture Organization of the UN, Rome.

Berti, M., R. Gesch, C. Eynck, J. Anderson, and S. Cermak. 2016. Camelina uses, genetics, genomics, production, and management. Ind. Crops Prod. 94:690-710. doi:10.1016/j.indcrop.2016.09.034

Berti, M., R. Gesch, B. Johnson, Y. Ji, W. Seames, and A. Aponte. 2015. Double-and relay-cropping of energy crops in the northern Great Plains, USA. Ind. Crops Prod. 75:26-34. doi:10.1016/j.indcrop.2015.05.012

Blackshaw, R., E. Johnson, Y. Gan, W. May, D. McAndrew, V. Barthet et al. 2011. Alternative oilseed crops for biodiesel feedstock on the Canadian prairies. Can. J. Plant Sci. 91:889-896. doi:10.4141/cjps2011-002

Budin, J.T., W.M. Breene, and D.H. Putnam. 1995. Some compositional properties of camelina (Camelina sativa L. Crantz) seeds and oils. J. Am. Oil Chem. Soc. 72:309-315.

Corporan, E., T. Edwards, L. Shafer, M.J. DeWitt, C. Klingshirn, S. Zabarnick et al. 2011. Chemical, thermal stability, seal swell, and emissions studies of alternative jet fuels. Energy Fuels 25:955-966. doi:10.1021/ ef101520v

Dilz, K. 1988. Efficiency of uptake and utilization of fertilizer nitrogen by plants. In: D.S. Jenkinson and K.A. Smith, editors, Nitrogen efficiency in agricultural soils. Elsevier Applied Science, London. p. 1-26.

French, A.N., D. Hunsaker, K. Thorp, and T. Clarke. 2009. Evapotranspiration over a camelina crop at Maricopa, Arizona. Ind. Crops Prod. 29:289-300. doi:10.1016/j.indcrop.2008.06.001

Gugel, R., and K. Falk. 2006. Agronomic and seed quality evaluation of Camelina sativa in western Canada. Can. J. Plant Sci. 86:1047-1058. doi:10.4141/P04-081

Hergert, G., J. Margheim, A. Pavlista, D. Martin, T. Isbell, and R. Supalla. 2016. Irrigation response and water productivity of deficit to fully irrigated spring camelina. Agric. Water Manage. 177:46-53. doi:10.1016/j. agwat.2016.06.009

Hunsaker, D.J., A.N. French, T.R. Clarke, and D.M. El-Shikha. 2011. Water use, crop coefficients, and irrigation management criteria for camelina production in arid regions. Irrig. Sci. 29:27-43. doi:10.1007/ s00271-010-0213-9
Hunsaker, D.J., A.N. French, and K.R. Thorp. 2013. Camelina water use and seed yield response to irrigation scheduling in an arid environment. Irrig. Sci. 31:911-929. doi:10.1007/s00271-012-0368-7

Isfan, D. 1990. Nitrogen physiological efficiency index in some selected spring barley cultivars. J. Plant Nutr. 13:907-914. doi:10.1080/01904169009364125

Jiang, Y.C.D., K.C. Caldwell, R.R. Falk, D. Lada, and D. MacDonald. 2013. Camelina yield and quality response to combined nitrogen and sulfur. Agron. J. 105:1847-1852. doi:10.2134/agronj2013.0240

Larsson, M. 2013. Cultivation and processing of Linum usitatissimum and Camelina sativa in southern Scandinavia during the Roman Iron Age. Veg. Hist. Archaeobot. 22:509-520. doi:10.1007/s00334-013-0413-3

Malhi, S., E. Johnson, L. Hall, W. May, S. Phelps, and B. Nybo. 2014. Effect of nitrogen fertilizer application on seed yield, $\mathrm{N}$ uptake, and seed quality of Camelina sativa. Can. J. Soil Sci. 94:35-47. doi:10.4141/cjss2012-086

Mohammed, Y.A., C. Chen, and R. Keshavarz-Afshar. 2017. Nutrient requirements of camelina for biodiesel feedstock in central Montana. Agron. J. 109:309-316. doi:10.2134/agronj2016.03.0163

Mon, J., K.F. Bronson, D.J. Hunsaker, K.R. Thorp, J.W. White, and A.N. French. 2016. Interactive effects of nitrogen fertilization and irrigation on grain yield, canopy temperature, and nitrogen use efficiency in overhead sprinkler-irrigated durum wheat. Field Crops Res. 191:54-65. doi:10.1016/j.fcr.2016.02.011

Novoa, R., and R.S. Loomis. 1981. Nitrogen and plant production. Plant Soil 58:177-204.

Pavlista, A., G. Hergert, J. Margheim, and T. Isbell. 2016. Growth of spring camelina (Camelina sativa) under deficit irrigation in western Nebraska. Ind. Crops Prod. 83:118-123. doi:10.1016/j.indcrop.2015.12.017

Pereira, L.S., I. Cordery, and I. Iacovides. 2012. Improved indicators of water use performance and productivity for sustainable water conservation and saving. Agric. Water Manage. 108(1):39-51. doi:10.1016/j. agwat.2011.08.022

Piepho, H.P., A. Büshcse, and C. Richter. 2004. A mixed modelling approach for randomized experiments with repeated measures. J. Agron. Crop Sci. 190:230-247. doi:10.1111/j.1439-037X.2004.00097.x

Putnam, D., J. Budin, L. Field, and W. Breene. 1993. Camelina: A promising low-input oilseed. In: J. Janick and J.E. Simon, editors, New crops. John Wiley \& Sons, New York. p. 314-322.

SAS Institute. 2013. The SAS system for Windows version 9.3. SAS Inst., Cary, NC.

Sivakumar, D., R. Sakthikumar, B. Raghunandan, J. Hu, S. Pruri, and A. Jain. 2015. Atomization characteristics of camelina-based alternative aviation fuels discharging from dual-orifice injector. J. Eng. Gas Turbines Power 137(8):081503. doi:10.1115/1.4029426

Urbaniak, S., C. Caldwell, V. Zheljazkov, R. Lada, and L. Luan. 2008. The effect of cultivar and applied nitrogen on the performance of Camelina sativa L. in the Maritime Provinces of Canada. Can. J. Plant Sci. 88:111119. doi:10.4141/CJPS07115

Vollmann, J., T. Moritz, C. Kargl, S. Baumgartner, and H. Wagentristl. 2007. Agronomicevaluation of camelina genotypes selected for seed quality characteristics. Ind. Crops Prod. 26:270-277. doi:10.1016/j. indcrop.2007.03.017

Witt, C., A. Dobermann, S. Abdulrachman, H.C. Gines, W. Guangho, R. Nagarajan et al. 1999. Internal nutrient efficiencies of irrigated lowland rice in tropical and subtropical Asia. Field Crops Res. 63:113-138. doi:10.1016/S0378-4290(99)00031-3

Wysocki, D.J., T.G. Chastain, W.F. Schillinger, S.O. Guy, and R.S. Karow. 2013. Camelina: Seed yield response to applied nitrogen and sulfur. Field Crops Res. 145:60-66. doi:10.1016/j.fcr.2013.02.009

Zubr, J. 1997. Oil-seed crop: Camelina sativa. Ind. Crops Prod. 6:113-119. doi:10.1016/S0926-6690(96)00203-8

Zubr, J. 2003. Qualitative variation of Camelina sativa seed from different locations. Ind. Crops Prod. 17:161-169. 